\title{
Patient-derived cancer organoid tracking with wide-field one-photon redox imaging to assess treatment response
}

\author{
Daniel A. Gil $\oplus^{\text {a,b }}$ Dustin Deming, ${ }^{\text {c,d,e,f }}$ and Melissa C. Skala $\odot^{\text {a,b,c,* } *}$ \\ ${ }^{a}$ University of Wisconsin, Department of Biomedical Engineering, Madison, Wisconsin, \\ United States \\ ${ }^{\mathrm{b}}$ Morgridge Institute for Research, Madison, Wisconsin, United States \\ c University of Wisconsin Carbone Cancer Center, Madison, Wisconsin, United States \\ ${ }^{\mathrm{d}}$ University of Wisconsin, Division of Hematology and Oncology, Department of Medicine, \\ Madison, Wisconsin, United States \\ ${ }^{e}$ University of Wisconsin, McArdle Laboratory for Cancer Research, Madison, Wisconsin, \\ United States \\ ${ }^{\mathrm{f}}$ William S. Middleton Memorial Veterans Hospital, Madison, Wisconsin, United States
}

\begin{abstract}
Significance: Accessible tools are needed for rapid, non-destructive imaging of patient-derived cancer organoid (PCO) treatment response to accelerate drug discovery and streamline treatment planning for individual patients.

Aim: To segment and track individual PCOs with wide-field one-photon redox imaging to extract morphological and metabolic variables of treatment response.

Approach: Redox imaging of the endogenous fluorophores, nicotinamide dinucleotide (NADH), nicotinamide dinucleotide phosphate (NADPH), and flavin adenine dinucleotide (FAD), was used to monitor the metabolic state and morphology of PCOs. Redox imaging was performed on a wide-field one-photon epifluorescence microscope to evaluate drug response in two colorectal PCO lines. An automated image analysis framework was developed to track PCOs across multiple time points over $48 \mathrm{~h}$. Variables quantified for each PCO captured metabolic and morphological response to drug treatment, including the optical redox ratio (ORR) and organoid area.

Results: The ORR (NAD(P)H/(FAD + NAD(P)H)) was independent of PCO morphology pretreatment. Drugs that induced cell death decreased the ORR and growth rate compared to control. Multivariate analysis of redox and morphology variables identified distinct PCO subpopulations. Single-organoid tracking improved sensitivity to drug treatment compared to pooled organoid analysis.

Conclusions: Wide-field one-photon redox imaging can monitor metabolic and morphological changes on a single organoid-level, providing an accessible, non-destructive tool to screen drugs in patient-matched samples.

(C) The Authors. Published by SPIE under a Creative Commons Attribution 4.0 Unported License. Distribution or reproduction of this work in whole or in part requires full attribution of the original publication, including its DOI. [DOI: 10.1117/1.JBO.26.3.036005]
\end{abstract}

Keywords: autofluorescence; redox imaging; image analysis; tracking; drug screening; cancer organoid.

Paper 200400R received Dec. 11, 2020; accepted for publication Feb. 24, 2021; published online Mar. 22, 2021.

\section{Introduction}

Precision medicine aims to improve cancer treatment by matching optimal therapies for each patient, typically based on genomic mutations. ${ }^{1-3}$ Although effective for a subset of mutations

*Address all correspondence to Melissa C. Skala, mcskala@wisc.edu 
(e.g., the genes $E G F R$ in lung cancer and $B R A F$ in melanoma), this approach has not been as successful in all cancers. ${ }^{4}$ Alternatively, drugs can be directly tested on a patient's tumor cells, providing functional information on drug sensitivity that is complementary to genomic approaches. ${ }^{4}$ Patient-derived cancer organoids (PCOs) are 3D organotypic cultures grown from fresh tumor samples (e.g., surgery and biopsy). PCOs recapitulate the in vivo molecular, histopathological, and phenotypic features of the original patient tumor. ${ }^{5-7}$ PCOs also provide accurate models of patient drug sensitivity, so that multiple drugs can be screened in patient-matched samples for streamlined drug development and clinical treatment planning. ${ }^{5,6,8-11}$ Additionally, PCOs capture the cellular heterogeneity found in tumors, which can result in treatment failure if drug resistant cell subpopulations are present. ${ }^{5,10,12,13}$ Therefore, it is critical to measure drug response across multiple PCOs to capture the response of subpopulations and accurately predict patient response. However, many tools to evaluate drug response in PCOs, such as single-cell RNA sequencing or histology, are destructive, providing only a static measurement that does not capture the dynamic nature of drug response and prohibits the use of complementary assays. ${ }^{14,15}$ This highlights a significant need to quantify drug response in PCOs using non-destructive tools that are sensitive to cellular heterogeneity.

Cellular metabolism is altered in cancer, and most cancer therapies disrupt cellular metabolism to limit proliferation or induce apoptosis. ${ }^{16}$ Autofluorescence can non-destructively monitor cellular metabolism within intact samples through the endogenous, metabolic co-enzymes nicotinamide dinucleotide (NADH), nicotinamide dinucleotide phosphate (NADPH), and flavin adenine dinucleotide (FAD). ${ }^{17-36} \mathrm{NADH}$ and NADPH have overlapping fluorescent properties and are jointly referred to as $\mathrm{NAD}(\mathrm{P}) \mathrm{H}$. $\mathrm{NAD}(\mathrm{P}) \mathrm{H}$ and $\mathrm{FAD}$ are involved in hundreds of metabolic reactions, including glycolysis and oxidative phosphorylation. The fluorescence intensities of NAD(P)H and FAD can be combined into an "optical redox ratio (ORR)" [NAD(P)H/(FAD + $\mathrm{NAD}(\mathrm{P}) \mathrm{H})]$ that provides a per-pixel map of the oxidation-reduction state of a sample. ${ }^{31,37,38}$ The ORR is sensitive to early drug-induced changes in cell metabolism that precede changes in tumor volume, proliferation (e.g., Ki67), and cell death (e.g., cleaved caspase 3). ${ }^{34,38-43}$ However, most studies of autofluorescence in PCOs have used expensive multiphoton and/or fluorescence lifetime imaging microscopes that are not widely available. ${ }^{34,38-43}$ A recent study by our group used selective plane illumination (light sheet) microscopy to image the autofluorescence of multiple PCOs, but required non-standard culture dishes to accommodate the imaging geometry of the microscope.$^{44}$ These drawbacks complicate the application of these techniques in drug discovery or the clinic. Disseminating autofluorescence screens beyond specialized laboratory use requires validation of accessible instrumentation that is compatible with standard cell culture methods.

Redox images can be collected with commonly available wide-field one-photon epifluorescence microscopes, which generally include a broadband excitation source, scientific monochrome camera, and standard filter cubes [4',6-diamidino-2-phenylindole (DAPI) and green fluorescent protein (GFP) filter cubes were used for $\mathrm{NAD}(\mathrm{P}) \mathrm{H}$ and $\mathrm{FAD}$, respectively]. ${ }^{17}$ Wide-field onephoton redox imaging has been previously applied at the cell and tissue level by our group and others to measure oxidative stress, mitochondrial dysfunction, cell phenotypes, apoptosis, and drug response. ${ }^{17,26-28,31-33,35,36,45}$ In this wide-field configuration, redox imaging can capture the ORR of each pixel along with the morphology of each PCO, but this configuration does not resolve individual cells as in previous two-photon redox imaging studies. ${ }^{34,38-43}$ However, wide-field one-photon redox imaging can rapidly image large populations of PCOs, which is important for characterizing subpopulations of PCOs with variable treatment sensitivities. ${ }^{9,46}$

Here we provide the first demonstration of wide-field one-photon redox imaging of PCOs. A image analysis framework was developed to automatically quantify organoid-level changes in redox ratio, autofluorescence intensity, and morphology over a treatment time course. This approach assessed the response to two chemotherapies (cisplatin and paclitaxel) and two metabolic inhibitors (sodium cyanide and 2-deoxy-glucose) across two colorectal PCO lines. The quantitative image analysis pipeline tracked drug response in each PCO before treatment and over multiple time points posttreatment up to $48 \mathrm{~h}$. Bivariate analysis revealed that morphological, redox ratio, and autofluorescence intensity measurements of drug response provide complementary information. Linear mixed-effect models were used to analyze these longitudinal organoid-level data, which provided improved sensitivity to drug response compared to conventional methods that pool organoid response over time (i.e., do not track individual PCO 
Gil, Deming, and Skala: Patient-derived cancer organoid tracking with wide-field one-photon...

response). A multivariate analysis of organoid-level redox ratio, autofluorescence intensity, and morphological variables showed distinct subpopulations of PCOs pretreatment, and heterogeneity in treatment-induced changes across PCOs. This work demonstrates that wide-field one-photon redox imaging is an accessible tool for monitoring changes in morphology and metabolism in PCOs, and that single-organoid tracking provides improved sensitivity to PCO drug response.

\section{Methods}

\subsection{Patient-Derived Cancer Organoid Culture}

PCOs were generated from two patient-derived metastatic colorectal cancer lines (P1 and P2), according to a previously published protocol. ${ }^{47}$ These lines were both derived from colorectal cancer metastases to the liver. Five wells (one well for each of the five treatment groups) were generated in each of the 24-well plates. The base culture medium used was DMEM/ F12 (ThermoFisher) supplemented with 10\% FBS (Sigma), and 1\% penicillin-streptomycin (Sigma). Briefly, previously grown PCOs were singularized with $0.25 \%$ trypsin, resuspended in base medium, and mixed with Matrigel (corning \# 354,234) at a 1:1 ratio. The cellMatrigel mixture was pipetted onto the glass surface of each well in a 24-well glass bottom plate (black frame, \#0 cover glass, Cellvis, P24-0-N). The plate was incubated at $37^{\circ} \mathrm{C}$ for 2 to $3 \mathrm{~min}$ to allow the mixture to solidify and then inverted to ensure PCOs were suspended in the Matrigel. The mixture was left to solidify for at least $20 \mathrm{~min}$, then base medium supplemented with Wnt3a-conditioned medium (1:1 ratio) in each well [Fig. 1(a), organoid culture]. Medium was refreshed every two days. Aberrant $\mathrm{Wnt} / \beta$-catenin signaling is a hallmark of colorectal cancer, and previous studies show that medium conditioned with Wnt3a supports colorectal PCO growth. ${ }^{48}$ Wnt3a-conditioned medium is generated by harvesting medium, in which murine L Wnt3a cells (ATCC, CRL-2647) have been cultured.

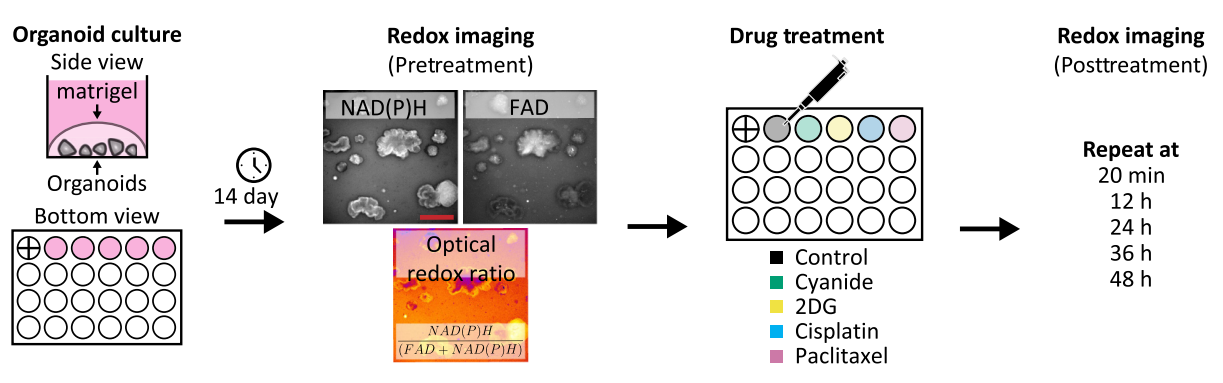

(a)
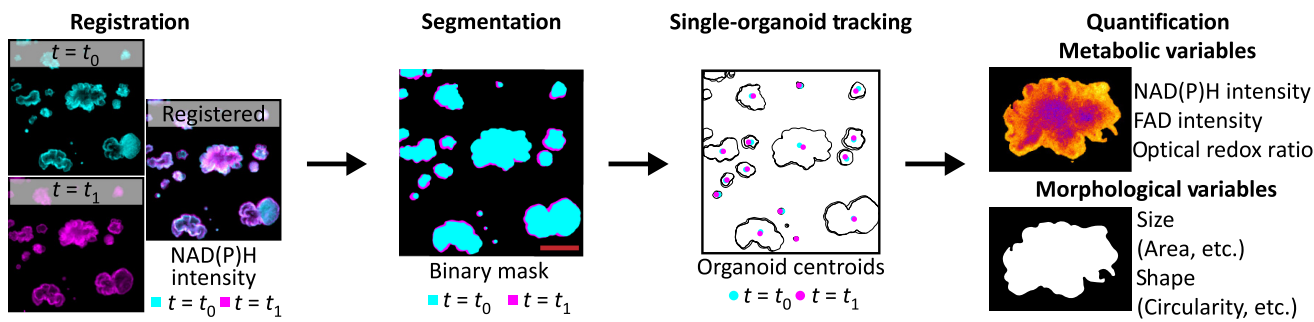

(b)

Fig. 1 Redox imaging of PCOs. An overview of the protocol for redox imaging and quantitative image analysis. (a) Graphical protocol showing the culturing of PCOs, pretreatment redox imaging, drug treatment, and posttreatment redox imaging time course. Redox imaging uses pairs of $\mathrm{NAD}(\mathrm{P}) \mathrm{H}$ and FAD fluorescence images from the same field-of-view to calculate the ORR image $[N A D(P) H /(F A D+N A D(P) H)]$. "+" in first well indicates registration mark. (b) Graphical representation of the image analysis pipeline, which includes registration of all frames in each image time series, organoid segmentation, single-organoid tracking, and quantification of metabolic and morphological variables to capture PCO drug response at each time point. Scale bar: $500 \mu \mathrm{m}$. 
Gil, Deming, and Skala: Patient-derived cancer organoid tracking with wide-field one-photon...

Table 1 Treatment groups, drug concentrations, and number of PCOs $(n)$ assessed for P1 and P2 lines.

\begin{tabular}{lccccc}
\hline \hline Treatment group & Drug class & Mechanism of action & Concentration & $n(\mathrm{P} 1)$ & $n(\mathrm{P} 2)$ \\
\hline Sodium cyanide $^{49}$ & Metabolic inhibitor & $\begin{array}{l}\text { Mitochondrial complex IV } \\
\text { inhibition }\end{array}$ & $4 \mathrm{mmol} / \mathrm{L}$ & 14 & 11 \\
$2 \mathrm{DG}^{50}$ & Metabolic inhibitor & $\begin{array}{l}\text { Glucose-6-phosphate } \\
\text { production inhibitor }\end{array}$ & $10 \mathrm{mmol} / \mathrm{L}$ & 13 & 8 \\
Cisplatin $^{51}$ & Chemotherapy & DNA replication inhibition & $33 \mu \mathrm{mol} / \mathrm{L}$ & 7 & 4 \\
Paclitaxel $^{52}$ & Chemotherapy & Microtubule stabilization & $0.5 \mu \mathrm{mol} / \mathrm{L}$ & 4 & 6 \\
$\begin{array}{l}\text { Control } \\
\text { (no treatment) }\end{array}$ & n/a & n/a & n/a & 10 & 6 \\
& & Total & 48 & 35 \\
\hline \hline
\end{tabular}

\subsection{Treatment Protocol}

Five wells for each PCO line were grown for 14 days before treatment. At day 14, media in the wells were refreshed with normal growth medium or medium containing sodium cyanide, 2-deoxy-glucose (2DG), cisplatin, or paclitaxel [Fig. 1(a), drug treatment]. Specifics of each treatment group, including the mechanism of action, concentration used, and number of PCOs per group, are listed in Table 1.

\subsection{Redox Imaging}

Redox imaging was performed using an inverted epifluorescence microscope (Nikon Ti-U). System specifications included a $4 \times$ air objective (Nikon CFI Plan Fluor, NA 0.13, FOV: $3.33 \mathrm{~mm} \times 3.33 \mathrm{~mm}$, lateral resolution: $2.16 \mu \mathrm{m}$ at $460 \mathrm{~nm} / 2.46 \mu \mathrm{m}$ at $525 \mathrm{~nm}$ ), a white light LED source (SOLA FISH, Lumencor), a scientific grade CMOS camera (Flash4, Hamamatsu, image pixel size: $1.625 \mu \mathrm{m}$ ), and an $X Y Z$ automated stage (MS-2000, ASI). Microscope control was performed with $\mu$ Manager. ${ }^{53} \mathrm{NAD}(\mathrm{P}) \mathrm{H}$ fluorescence was excited with a DAPI filter cube (Nikon, ex: 361 to $389 \mathrm{~nm} / \mathrm{em}$ : 435 to $485 \mathrm{~nm}$ ) and integrated over $3 \mathrm{~s}$. FAD fluorescence was excited with a GFP filter cube (Nikon, ex: 450 to $490 \mathrm{~nm} / \mathrm{em}$ : 500 to $550 \mathrm{~nm}$ ) and integrated over $5 \mathrm{~s}$.

An NAD $(\mathrm{P}) \mathrm{H}$ fluorescence image and FAD fluorescence image were acquired for each well pretreatment and at $20 \mathrm{~min}, 12,24,36$, and $48 \mathrm{~h}$ posttreatment [Fig. 1(a), redox imaging]. The $\mathrm{NAD}(\mathrm{P}) \mathrm{H}$ and FAD images were used to calculate the 2D ORR for each pixel position $(i, j)$ using the following equation:

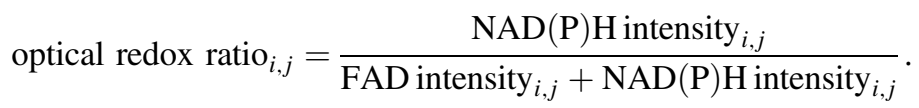

The entire imaging protocol including $X Y Z$ stage repositioning and image acquisition was automated with $\mu$ Manager. For each plate, a total of five wells were imaged. Total imaging time per plate was $<2$ min, capturing 48 PCOs across five wells for P1 and 35 PCOs across five wells for P2. Note that the first well of the 24-well plate was used to assist in positioning the plate before imaging, which enabled reproducible measurements of the same field-of-view over multiple time points [Fig. 2(a)].

\subsection{Quantitative Image Analysis}

A framework for redox imaging and automated image analysis was developed to track each PCO across multiple time points and quantify organoid metabolism and morphology [Fig. 1(b)]. 
Gil, Deming, and Skala: Patient-derived cancer organoid tracking with wide-field one-photon...

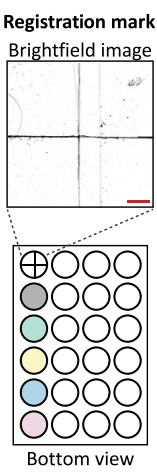

(a)

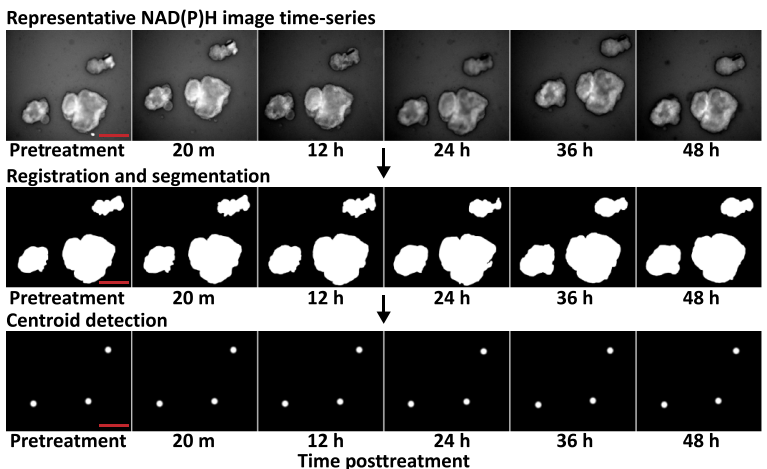

(b)

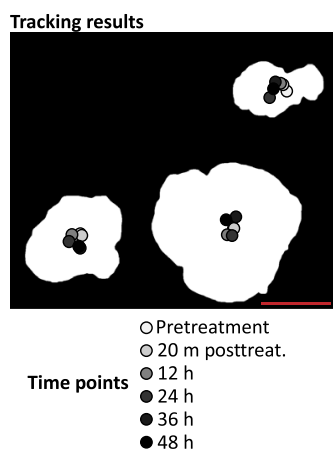

(c)

Fig. 2 Single-organoid tracking. Organoid tracking over multiple time points was achieved with a registration mark and single-particle tracking. (a) A registration mark was inscribed on the bottom of the first well of the 24-well plate and referenced at each time point to ensure accurate $X Y Z$ positioning. (b) Organoid tracking was achieved by registering the $N A D(P) H$ image time series, segmenting the organoids in each frame, and detecting the centroids of each segmented organoid. These centroids were tracked using an open-source single-particle tracking tool (TrackMate). (c) A segmented organoid mask at 48-h posttreatment is overlaid with the centroid tracking results at each imaging time point. Scale bar: $500 \mu \mathrm{m}$.

Linkage of each organoid over time was performed via a combined approach of NAD(P)H image time series registration and a $2 \mathrm{D}$ centroid matching-based approach with single-particle tracking. This pipeline was implemented using MATLAB (2019b, Mathworks), except when specifically noted.

Registration of each image time series was achieved using both a registration mark and rigid image registration [Figs. 2(a) and 2(b)]. A registration mark was etched on the glass bottom of the first well of each plate using a diamond-tip scribe and was referenced at every time point to precisely position the plate before imaging to minimize $X Y Z$ drift [Fig. 2(a)]. After acquisition, $X Y$ drift between frames was corrected through rigid image registration, which registers two frames by maximizing the cross correlation of pixel values.$^{54,55}$ The $\mathrm{NAD}(\mathrm{P}) \mathrm{H}$ image time series was used to calculate the $X Y$ shift values needed to align the frames in the $\mathrm{NAD}(\mathrm{P}) \mathrm{H}$ image time series, and these shift values were applied to the corresponding FAD and ORR image time series. These shift values were also used to determine areas that are common across all frames. Image areas that did not overlap across the image time series were excluded.

Segmentation of the PCOs was independently performed on the $\mathrm{NAD}(\mathrm{P}) \mathrm{H}$ image at each time point to capture changes in organoid size and shape [Fig. 2(b)]. However, segmentation based on intensity alone is challenging because autofluorescence images have a low signalto-background ratio (typically between 2 and 3). A segmentation algorithm based on edge enhancement was developed using MATLAB that reliably segmented all PCOs imaged in this study. Algorithm 1 is as follows, below.

Specific values for algorithm parameters were chosen empirically based on visual inspection. Validation of the segmentation algorithm was performed by calculating the Sørenson-Dice similarity coefficient for pairs of automatically segmented images and manual segmented images (Fig. S1 in the Supplemental Materials, $n=5$, mean $\pm \mathrm{SEM}=0.93 \pm 0.05$ ).

Tracking of each PCO was achieved using an open-source single-particle tracking tool (TrackMate, ImageJ/FIJI). ${ }^{57}$ TrackMate achieves single-particle tracking by detecting particles in each frame, linking detected particles from frame-to-frame, and combining all links into the most likely tracks. ${ }^{58}$ Image time series were generated using MATLAB with 2D Gaussian particles (kernel: $10 \times 10$ pixels) at the centroid of each segmented PCO [Fig. 2(b), centroid detection]. These centroids were then identified in each frame using the difference-of-Gaussian blob detector (options: 10 pixel diameter, no spot filtering). The linear assignment problem algorithm was chosen to perform particle tracking because it is sensitive to events such as particle drift, particle merging, or over-/under-segmentation (options: 200 pixel maximum linking distance, 200 pixel max gap-closing distance, and 2 frame max gap-closing distance). All key parameters 
Algorithm 1 Organoid Segmentation

1. Median filter was applied to remove noise (medfilt2, kernel size: $25 \times 25$ pixels).

2. Gaussian filter was applied to estimate the background, and this background was subtracted from the image (imgaussfilt, kernel size: $450 \times 450$ pixels).

3. Local standard deviation filter was applied to enhance edges (stdfilt, kernel: $13 \times 13$ pixels).

4. Otsu's method was used to segment the edge-enhanced image into three classes (imquantize, multithresh, and number of threshold values: (2). The lowest class, corresponding to the non-organoid regions, was excluded.

5. Segmented regions less than 100 pixels were removed.

6. Holes in segmented regions were filled in (imfill, option: "holes").

7. Segmented regions were eroded with a disk structure element (imerode, strel, options: "disk," 9 pixels).

8. Active contours refined the segmented regions (activecontours, options: "Chan-Vese," 200 iterations, -0.6 contraction bias).

9. Separation of touching organoids in the mask was achieved via the standard h-Minima-watershed transform algorithm.

10. Holes in segmented regions were filled-in (imfill, option: "holes").

11. Edges in segmented regions were smoothed with a Gaussian filter (imgaussfilt, kernel size: $5 \times 5$ pixels).

12. Segmented regions corresponding to PCOs were filtered based on morphology (regionprops, ismember, options: regions with area $>1000$ pixels and circularity $>0.4) .{ }^{56}$

were initially chosen by visual inspection of the tracking results for the P1 control time series and then applied to all other image time series [Fig. 2(c)]. Once completed for an image time series, the single-organoid tracking results were used to link the metabolic and morphological variables for each organoid over time.

Quantification of variables that describe organoid morphology and metabolism was performed for each PCO at every time point ( $n=83$ PCOs total, $n=48$ PCOs for P1, and $n=$ 35 PCOs for P2). In total, 24 variables (12 metabolic and 12 morphological) were quantified for each segmented organoid: mean, minimum, maximum, and standard deviation of the ORR, $\mathrm{NAD}(\mathrm{P}) \mathrm{H}$ intensity, and FAD intensity values; organoid area, perimeter, solidity, extent, eccentricity, circularity, minimum Feret's diameter, maximum Feret's diameter, minor axis, major axis, convex area, and equivalent diameter (regionprops). To limit the effects of variation in the culture medium autofluorescence, no background subtraction was performed on the images before quantification. Detailed definitions of these variables are provided in Table S1 in the Supplemental Materials. PCOs connected to the image border were excluded from statistical analysis.

\subsection{Statistics}

All statistical analyses were performed using MATLAB or R. Open-source MATLAB toolboxes used were Gramm, a data visualization and statistics package, and drtoolbox, a dimensionality reduction package. ${ }^{59,60}$ Open-source $\mathrm{R}$ toolboxes included nlme, a mixed effect modeling package, and lsmeans, a least-squares means estimation package. ${ }^{61,62}$

Linear regression was also used to assess the bivariate relationship between organoid area and $\mathrm{ORR}, \mathrm{NAD}(\mathrm{P}) \mathrm{H}$ intensity, and FAD intensity pretreatment (stat_glm and Gramm). ${ }^{60}$ The linear pairwise correlations were also calculated for all 24 variables pretreatment (corr).

Organoid-level-normalized time series were calculated for each organoid as $X_{\text {posttreatment }} /$ $X_{\text {pretreatment organoid, }}$, where $X$ is the mean ORR or organoid area. Linear mixed-effect models were used to analyze these organoid-level-normalized time series for each condition and PCO line (nlme, R). ${ }^{61}$ Linear mixed-effect models can account for organoid-level differences in 
measurements using a random-intercept model, which provides a way to assess treatmentinduced effects in the presence of organoid-level heterogeneity. ${ }^{63,64} \mathrm{~A}$ random-intercept model was specified as $Y \sim$ time + treatment + treatment $*$ time $+(1$ organoid $)+$ error, where $Y$ is either the pretreatment-normalized ORR or organoid area, time is a categorical variable encoding time, treatment is a categorical variable encoding the five treatment groups, organoid is the organoid-level-normalized value at 20-min posttreatment, and error is the random error in the model. Time is defined as a categorical variable to avoid the assumption of linearity between time and the ORR or organoid area. Organoid here is encoded as a random effect ("1lorganoid" in the statistical model notation) to account for variability at the organoid level. Pretreatment values were excluded from the analysis because all time points were normalized to pretreatment values (i.e., pretreatment variance is one). Thus the first time point included in the analysis is 20-min posttreatment. A first-order autoregressive [i.e., AR(1)] covariance structure was used to account for the correlations found in these time-course data, where the correlation between time points decreases with increasing separation in time. Least-squares means was then used to calculate the pairwise differences between all treatment groups at each time point, compute the $p$-value for each pairwise difference using Tukey's "honest significant difference" method, and account for multiple comparisons (lsmeans, R) ${ }^{62}$

Comparison between single-organoid tracking and pooled analysis was performed. The pooled analysis was the same as the single-organoid tracking analysis, except for the normalization approach and statistical model. To mimic pooled organoid data without organoid tracking, data for each organoid was normalized to the well-level pretreatment mean as

$X_{\text {posttreatment }} / X_{\text {pretreatment well }}$, where $X$ is the mean ORR or organoid area. A fixed effects model was specified as $Y \sim$ time + treatment + treatment $*$ time + error, where $Y$ is either the welllevel-normalized ORR or organoid area, time is a categorical variable encoding time, treatment is a categorical variable encoding the five treatment groups, and error is the random error in the model. Pretreatment values were also not included in the analysis. All other parts of the pooled analysis were the same as the organoid-level analysis including the AR(1) covariance structure, least-squares mean for pairwise comparisons, and correction for multiple comparisons.

Principal component analysis (PCA) was used to explore the multivariate relationships between the 24 variables measured from each PCO. PCA was performed using an open-source MATLAB toolbox for dimensionality reduction (compute_mapping, out_of_sample, and drtoolbox). Data from all PCOs pretreatment, 24-h posttreatment, and 48-h posttreatment were standardized within each time point before PCA to account for differences in the scale of each variable (zscore). The loadings that projected the data into the principal component (PC) basis were computed using the data from all PCOs pretreatment. These loadings were then applied to data at 24-h and 48-h posttreatment to visualize the time-dependent effects of treatment on the variables from each PCO. Loading vectors were plotted to visualize the contribution of each variable to the PCs. For clarity, only the top 12 variables that loaded on PC1 and PC 2 were displayed as they are the variables that contributed the most variability. The projected data were scaled to fit in the loadings interval (biplot). The scaled scores of the data projected on the first two PCs were plotted and fitted with ellipses (i.e., bivariate normal distribution) that contained $95 \%$ of the points for each group (geom_point, stat_ellipse, and Gramm).

\section{Results}

\subsection{Optical Redox Ratio is Independent of Organoid Morphology}

The goal of this study was to segment and track individual PCOs with wide-field one-photon redox imaging to extract morphological and metabolic variables of drug response. A quantitative image analysis framework was developed to automatically quantify 12 metabolic variables that summarize $\mathrm{NAD}(\mathrm{P}) \mathrm{H}$ intensity, FAD intensity, and $\mathrm{ORR}$, along with 12 morphological variables that quantify organoid size and shape (Table S1 in the Supplemental Materials), for each organoid over time. Relationships between the area of a PCO and its mean NAD(P)H intensity, FAD intensity, and ORR are shown in Figs. 3(a)-3(c). Linear models were fit to paired measurements from all PCO pretreatment ( $n=48$ PCOs for P1, $n=35$ PCOs for P2). There is no correlation 


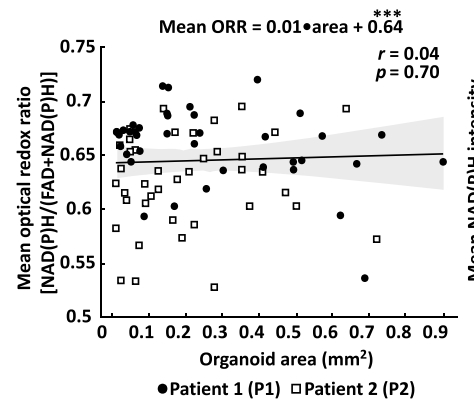

(a)

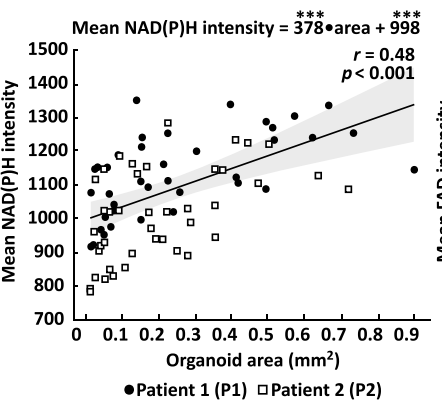

(b)

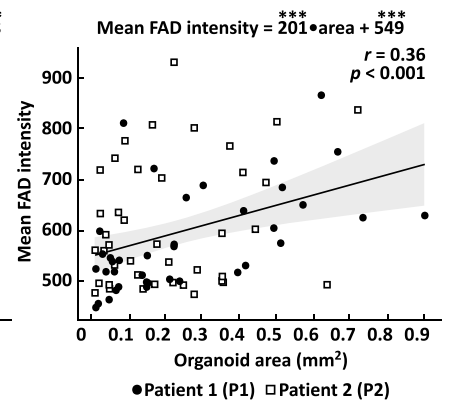

(c)

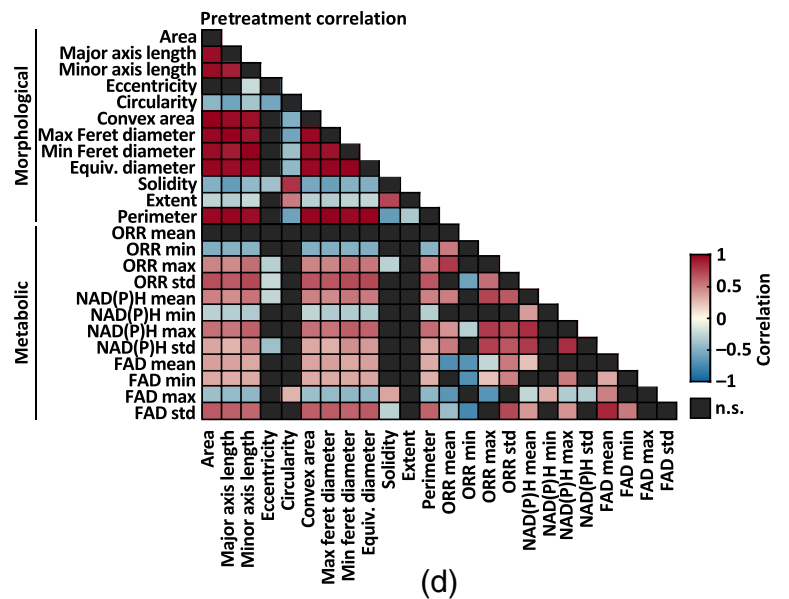

(d)

Fig. 3 Analysis of organoid-level redox imaging variables. Analysis of variables extracted from all PCOs pretreatment. (a) Linear regression was used to quantify the relationship between organoid area and its mean ORR. The fitted model indicates the mean ORR value of each PCO is independent of its area $(r=0.04, p=0.70)$ for all PCOs from patient 1 (P1) and patient (P2) pretreatment. Statistical significance of the model coefficients is indicated as ${ }^{\star \star *}$ for $p<0.001$. (b) Linear regression was used to quantify the relationship between organoid area and its mean $N A D(P) H$ intensity. The fitted model indicates a moderate positive correlation $(r=0.48, p<0.001)$ between organoid area and mean $\mathrm{NAD}(\mathrm{P}) \mathrm{H}$ intensity. (c) Linear regression was used to quantify the relationship between organoid area and its mean FAD intensity. The fitted model indicates a moderate positive correlation $(r=0.36, p<0.001)$ between organoid area and mean FAD intensity. (d) Linear pairwise correlation (Pearson's $r$ ) matrix of the variables extracted from each PCOs. Heatmap shows only statistically significant correlations $(p<0.05)$; n.s., not significant ( $p>0.05$, black boxes). Table 1 shows the number of PCOs in each treatment group for each patient.

between the mean ORR of each PCO and its area [Fig. 3(a), $r=0.04, p=0.70$ ]. However, there is a moderate positive correlation between organoid area and mean NAD $(\mathrm{P}) \mathrm{H}$ intensity [Fig. 3(b), $r=0.48, p<0.001]$ and between organoid area and mean FAD intensity [Fig. 3(c), $r=0.36, p<0.001]$.

The linear pairwise correlations of all 24 variables were computed to understand the relationships between the metabolic and morphological variables [Fig. 3(d)]. As expected, morphological variables were significantly correlated $(p<0.05)$ with most other morphological variables apart from eccentricity, which was only significantly correlated with the minor axis length, solidity, and circularity. Likewise, metabolic variables were significantly correlated $(p<0.05)$ with many other metabolic variables except for the minimum and maximum values, which were uncorrelated with many of the other metabolic variables. Statistically significant correlations between morphological and metabolic variables were also observed, especially for standard deviations in FAD intensity and ORR that positively correlate with greater organoid area/ diameter/length. Consistent with the linear model [Fig. 3(a)], the mean ORR had no significant correlations with any morphological parameters. Previous studies have shown that the ORR of 
cancer organoids predicts treatment response, ${ }^{9-12}$ so these data indicate that the mean ORR may provide information about PCO drug response that is independent of morphology.

\subsection{Drug Response Lowers Optical Redox Ratio}

Changes in the ORR were calculated in two PCO lines over a 48-h treatment time course. Previous studies have found that a treatment-induced decrease in the ORR of cancer organoids predicts in vivo treatment response. ${ }^{25,40}$ Treatment-induced changes in mean NAD(P)H intensity and mean FAD intensity can be found in Fig. S2(A)-(D) in the Supplemental Materials, which reflects the trends in the ORR time series. Both PCO lines were treated with cisplatin and paclitaxel, two chemotherapies, and sodium cyanide and 2DG, two metabolic inhibitors. There was one well for each treatment and an additional untreated well served as a negative control (five wells total). Three representative ORR image time series are shown in Fig. 4(a), which show the change in ORR with cyanide treatment, large change in ORR with paclitaxel treatment, and the

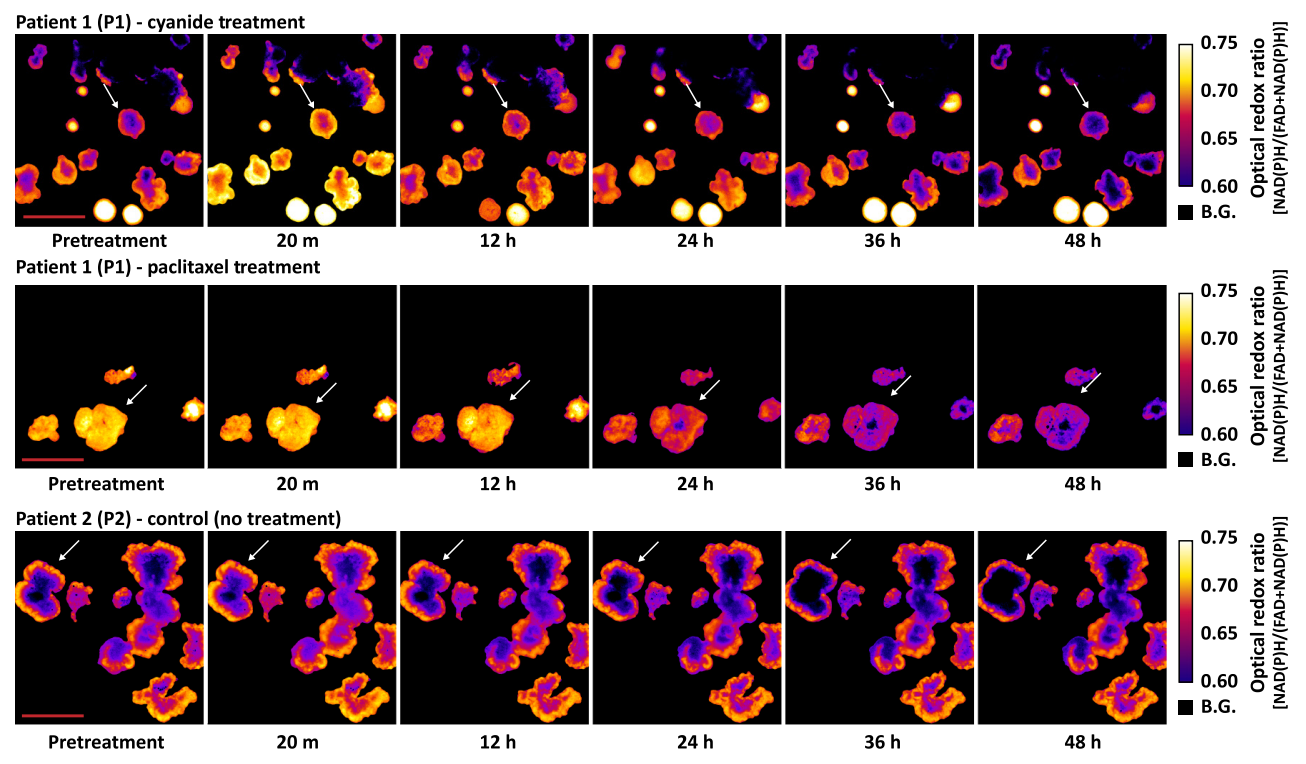

(a)

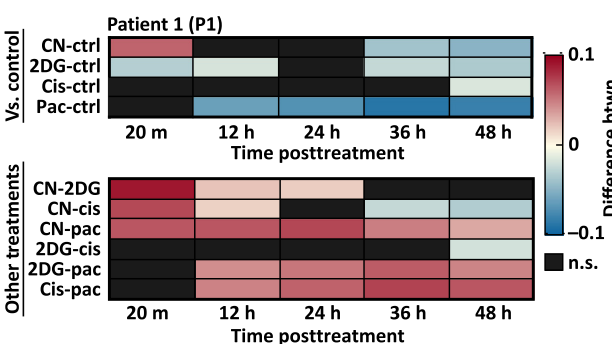

(b)

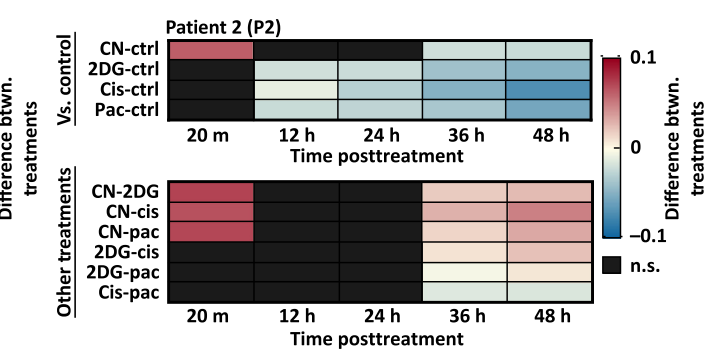

(c)

Fig. 4 Treatment-induced changes in PCO ORR. Quantitative image analysis tracked changes in the ORR of each PCO over time. (a) Representative ORR image time series for patient 1 (P1) and patient 2 (P2). Arrows indicate specific organoids representative of changes observed for that condition. B.G, background. (b) Heatmaps show the pairwise percent differences between pretreatment-normalized ORR between all treatment groups for $P 1$. Top: pairwise percent differences between each treatment and control for P1. Bottom: pairwise percent differences between drug treatments (excluding control) for P1. (c) Heatmaps show the pairwise percent differences between pretreatment-normalized ORR between all treatment groups for P2. Top: pairwise percent differences between each treatment and control for P2. Bottom: pairwise percent differences between drug treatments for P2. All pairwise differences were calculated from the linear mixedeffect models via least-squares means. n.s., not significant ( $p>0.05$, black boxes). Table 1 shows the number of PCOs in each treatment group for each patient. Scale bar: $500 \mu \mathrm{m}$. 
stability of the ORR in untreated organoids. Patient 1 PCOs treated with cyanide and paclitaxel were representative of a minimal and large response at 48-h posttreatment, respectively. Arrows in Fig. 4(a) indicate specific PCOs that are representative of changes observed for that condition. Single-organoid tracking was used to normalize each PCO to its pretreatment ORR, so that relative changes in ORR could be compared across PCOs. Least-squares means was used to estimate the mean of each group and to calculate the pairwise differences at each time point between treatment groups [Figs. 4(b) and 4(c)].

For P1 PCOs, the ORR decreased compared to control at $36 \mathrm{~h}$ for the cyanide $(p<0.01)$ and 2DG $(p<0.01)$ groups [Fig. 4(b)]. Cisplatin treatment in P1 resulted in a decreased ORR compared to control only at $48 \mathrm{~h}$ [Fig. 4(b), $p<0.05]$. Paclitaxel treatment in P1 resulted in a decreased ORR compared to control starting at $12 \mathrm{~h}$ [Fig. 4(b), $p<0.05$ ]. For P2 PCOs, the ORR decreased compared to control at $12 \mathrm{~h}$ for the 2DG $(p<0.01)$, cisplatin $(p<0.05)$, and paclitaxel $(p<0.05)$ groups [Fig. 4(c)]. Cyanide treatment in P2 resulted in a decreased ORR compared to control starting at $36 \mathrm{~h}$ [Fig. 4(c), $p<0.01$ ]. Additionally, cyanide in both P1 and P2 caused a sharp rise in the ORR $(p<0.001)$ at $20 \mathrm{~min}$, characteristic of electron transport chain inhibition [Figs. 4(b) and 4(c)]. Heatmaps [Figs. 4(b) and 4(c)] also show the pairwise differences between treatment groups.

\subsection{Drug Response Slows Organoid Growth}

Changes in organoid size or growth rate can occur due to effective drug response, so the relative change in organoid area over time was also quantified. All other morphological variables remained constant or had a limited change over $48 \mathrm{~h}$ compared to pretreatment values [a subset can be found in Fig. S2(E)-(J) in the Supplemental Materials]. Three representative image time series in Fig. 5(a) show segmented PCOs with the color-coded value mapped to the pretreatment normalized organoid area. These image time series illustrate increased organoid area over time in control conditions and relatively modest increases in organoid area over time in cyanide and paclitaxel treatment conditions. Arrows in Fig. 5(a) indicate specific PCOs representative of changes observed for that condition. Heterogeneity in organoid area was observed pretreatment, so single-organoid tracking was used to normalize each organoid to its pretreatment area. This enabled comparisons of relative changes in area across organoids. Least-squares means was used to estimate the mean of each group and to calculate the pairwise differences at each time point between control and treatment groups [Figs. 5(b) and 5(c)].

Two separate patterns of response emerged for metabolic inhibitors and chemotherapies. For P1 groups treated with metabolic inhibitors, lower organoid areas compared to control were observed starting at $12 \mathrm{~h}$ for the cyanide $(p<0.01)$ and 2DG $(p<0.01)$ [Fig. 5(b)]. However, paclitaxel-treated P1 PCOs continued to grow until $24 \mathrm{~h}$ and were only significantly different from control beginning at $36 \mathrm{~h}(p<0.05)$ [Fig. 5(b)]. For P1 PCOs, cisplatin treatment resulted in lower organoid areas compared to control only at $48 \mathrm{~h}(p<0.05)$ [Fig. 5(b)]. For P2 PCOs treated with metabolic inhibitors, lower organoid areas compared to control were observed starting at $12 \mathrm{~h}$ for the cyanide $(p<0.01)$ and 2DG $(p<0.01)$ groups [Fig. 5(c)]. For P2 PCOs treated with chemotherapies, continued growth was observed until $24 \mathrm{~h}$, and organoid area was only lower than control beginning at $36 \mathrm{~h}$ for paclitaxel $(p<0.01)$ and cisplatin $(p<0.01)$ groups [Fig. 5(c)]. Heatmaps [Figs. 5(b) and 5(c)] also show the pairwise differences between treatment groups.

\subsection{Single-Organoid Tracking Provides Improved Sensitivity}

PCOs are heterogeneous and exhibited a range of ORR and organoid area values. Singleorganoid tracking can monitor dynamic changes in organoid-level variables over time by normalizing each PCO to its own pretreatment value. This enabled the observation of the ORR and organoid area on an organoid-level in the P1 and P2 control groups. In both P1 and P2 control groups, the ORR was stable over $48 \mathrm{~h}$ [Figs. 6(a) and 6(c), $p>0.05$ pretreatment versus each time-point posttreatment using organoid-level normalization]. In both P1 and P2 control groups, PCOs continue to grow over $48 \mathrm{~h}$, with a statistically significant increase in organoid area of $10 \%$ 

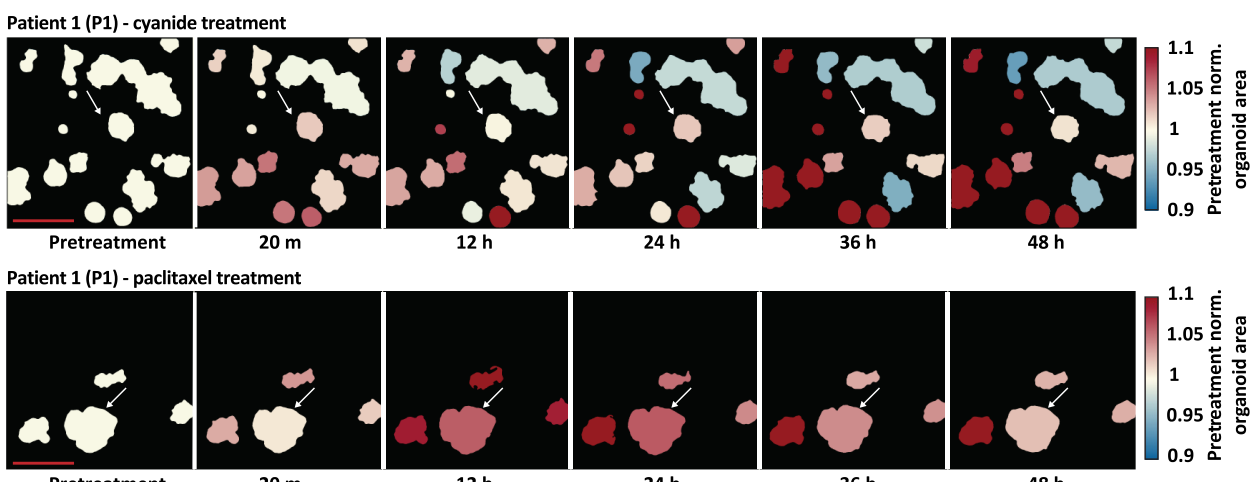

Pretreatment 2 (P2) - control (no treatment)
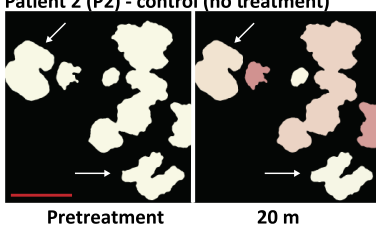

$12 \mathrm{~h}$

$24 \mathrm{~h}$

$36 \mathrm{~h}$
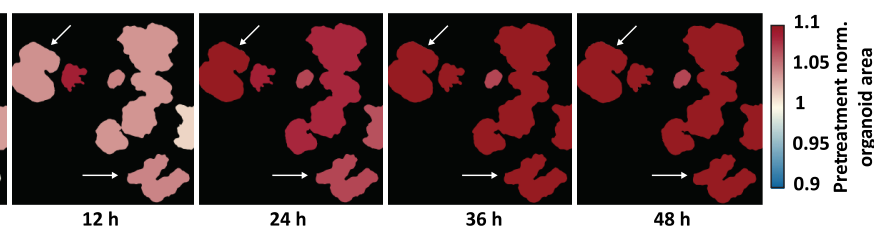

(a)

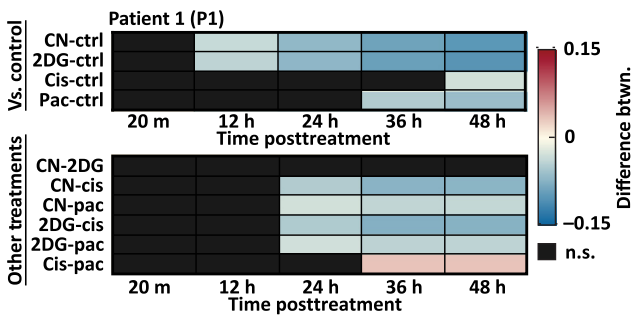

(b)

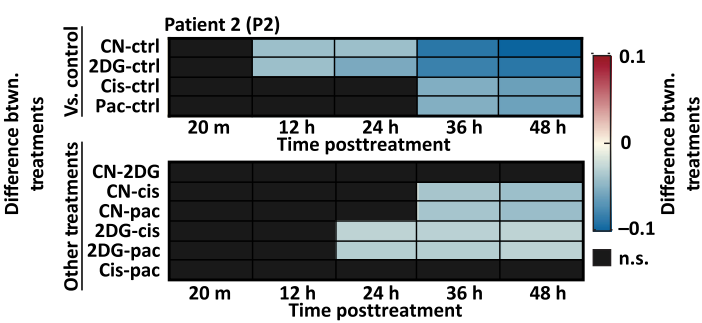

(c)

Fig. 5 Treatment-induced changes in PCO areas. Quantitative image analysis tracked changes in the area of each PCO over time. (a) Representative segmented image time series for patient 1 (P1) and patient 2 (P2). The color-coded value of each segmented organoid corresponds to value at each time point divided by its pretreatment value. Arrows indicate specific organoids that are representative of changes observed for that condition. (b) Heatmaps show the pairwise percent differences between pretreatment-normalized area between all treatment groups for P1. Top: pairwise percent differences between each treatment and control for $\mathrm{P} 1$. Bottom: pairwise percent differences between drug treatments (excluding control) for P1. (C) Heatmaps show the pairwise percent differences between pretreatment-normalized area between all treatment groups for P2. Top: pairwise percent differences between each treatment and control for P2. Bottom: pairwise percent differences between drug treatments for P2. All pairwise differences were calculated from the linear mixed-effect models via least-squares means. n.s., not significant $(p>0.05$, black boxes). Table 1 shows the number of PCO in each treatment group for each patient. Scale bar: $500 \mu \mathrm{m}$.

and $11 \%$, respectively, at $48 \mathrm{~h}$ compared to pretreatment [Figs. $6(\mathrm{e})$ and $6(\mathrm{~g}), p<0.05$ pretreatment versus each time-point posttreatment using organoid-level normalization].

To demonstrate the utility of single-organoid tracking, a pooled analysis was performed. In addition to the previous analysis using organoid-level normalization, data were analyzed using well-level normalization where each PCO was normalized to the well-level mean. Figure 6 shows the ORR and organoid area over time for P1 and P2 analyzed using single-organoid tracking (organoid-level normalization) or pooled analysis (well-level normalization). Singleorganoid tracking [Figs. 6(a), 6(c), 6(e), and 6(g)] provided data with lower variability compared to pooled analysis [Figs. 6(b), 6(d), 6(f), and 6(h)], as the well-level mean does not fully account for organoid-level heterogeneity. In addition, pooled analysis does not provide organoid-level time series for use in a statistical model. Single-organoid tracking found more differences between control and treatment groups when compared to pooled analysis, which demonstrates the improved sensitivity to treatment response afforded by single-organoid tracking. 


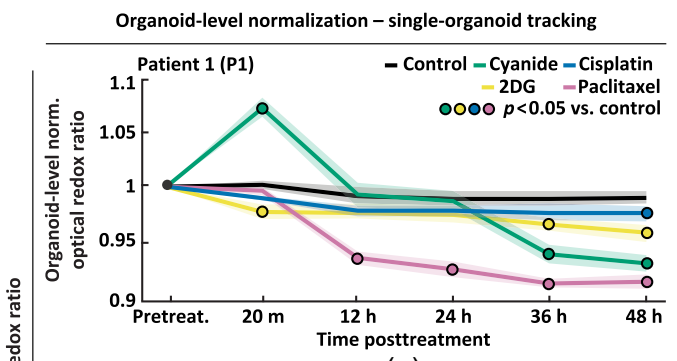

(a)

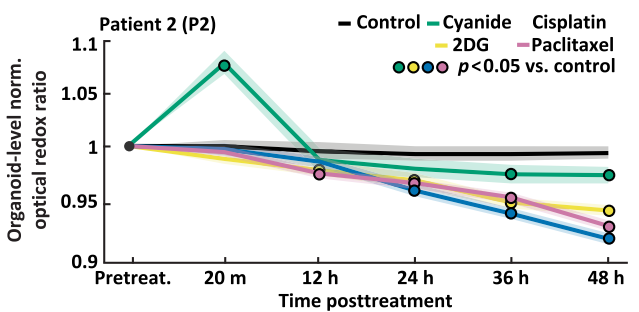

(c)

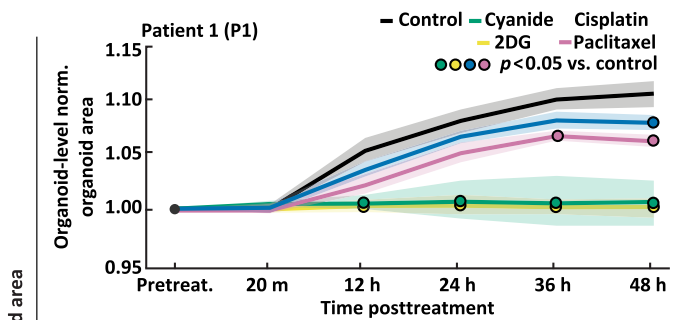

(e)

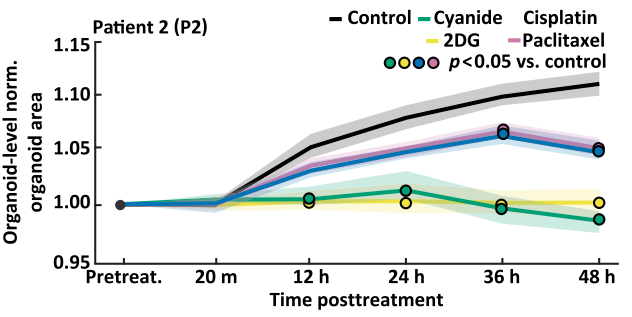

(g)

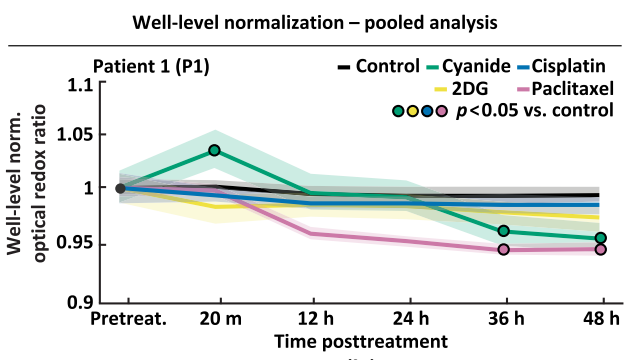

(b)

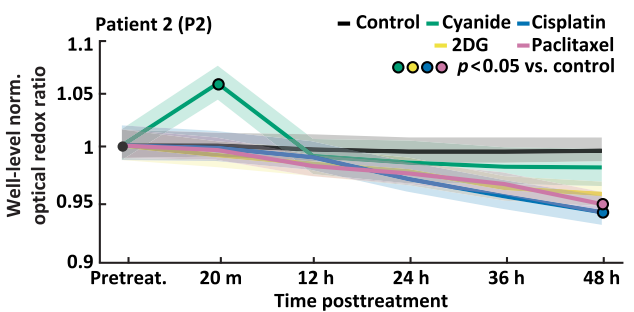

(d)

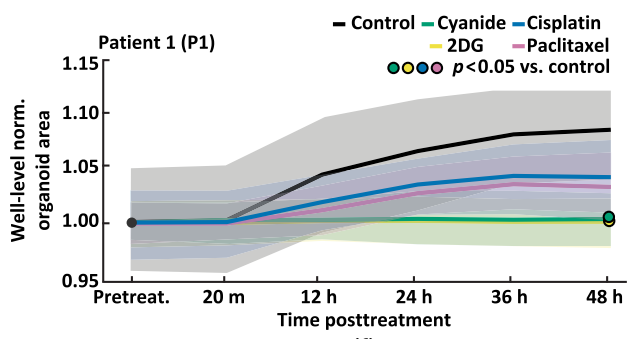

(f)

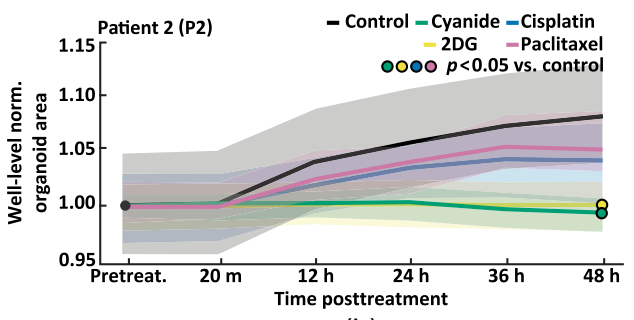

(h)

Fig. 6 Comparison of single-organoid tracking and pooled analysis. Comparison of singleorganoid tracking and pooled analysis using time series data from patient 1 (P1) and patient 2 (P2). Normalization refers to dividing the value of each organoid at each time point by either its own pretreatment value (organoid-level normalization) or the well-level mean (well-level normalization). (a), (b) Pretreatment normalized ORR using organoid-level normalization (left) and welllevel normalization (right) for each treatment group for P1. (c), (d) Pretreatment normalized ORR using organoid-level normalization (left) and well-level normalization (right) for each treatment group for P2. (e), (f) Pretreatment normalized organoid area using organoid-level normalization (left) and well-level normalization (right) for each treatment group for P1. (g), (h) Pretreatment normalized organoid area using organoid-level normalization (left) and well-level normalization (right) for each treatment group for P2. Data in (a), (c), (e), (g) were analyzed using a linear mixed-effect model that uses single-organoid tracking to account for organoid-level variability. Data in (b), (d), (f), (h) were analyzed using a linear mixed-effect model that does not account for organoid-level variability due to the lack of single-organoid tracking. Data plotted are mean (lines) \pm standard error of the mean (shaded area). Significant differences between a treatment and control $(p<0.05)$ are indicated with a circle color-coded for the treatment group (see legend). Table 1 shows the number of PCOs in each group and for each patient. 
Gil, Deming, and Skala: Patient-derived cancer organoid tracking with wide-field one-photon...

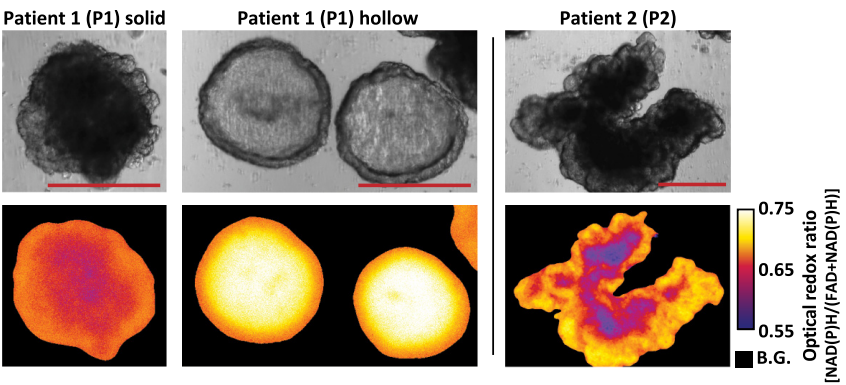

(a)

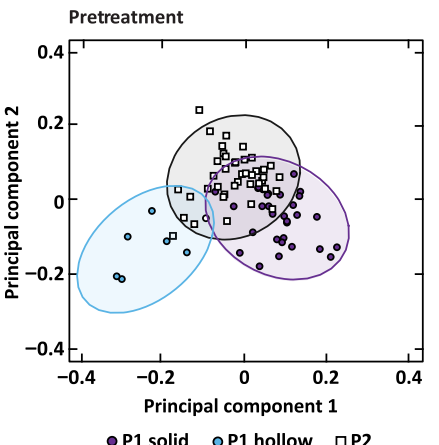

(c)

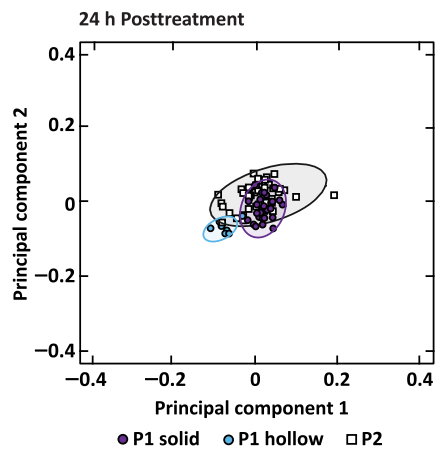

(d)

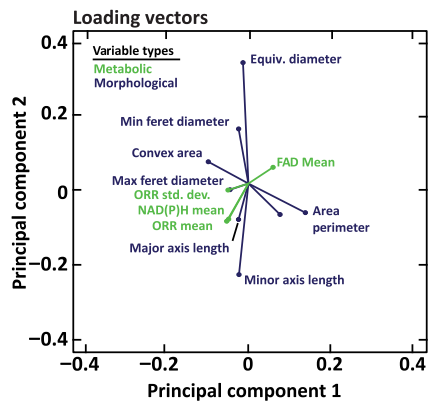

(b)

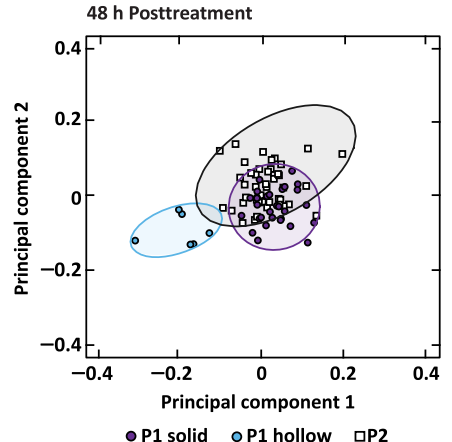

(e)

Fig. 7 Phenotypic identification of PCO subpopulations. Qualitative and quantitative (PCA) evaluation of PCOs with distinct phenotypes from patient 1 (P1) and patient 2 (P2). (a) Paired brightfield (top) and ORR (bottom) images of representative PCO from each of the three phenotypic subpopulations: P1 solid, P1 hollow, and P2. Scale bar: $250 \mu \mathrm{m}$. B.G., background. (b) Loading vectors for the 12 variables with the highest loading on PC1 and PC2, which were defined with pretreatment data only. Green are metabolic variables and blue are morphological variables. (c) Data from all organoids projected onto PC1 and PC2. Colors correspond to the three qualitatively identified phenotypic organoid subpopulations: P1 solid, P1 hollow, and P2. Ellipse containing $95 \%$ of the points for each group were also plotted. (d) The pretreatment PCs applied to data from the PCOs at 24-h posttreatment. (e) The pretreatment PCs applied to data from PCOs at 48-h posttreatment.

\subsection{Multivariate Analysis Reveals Patient-Derived Cancer Organoid Subpopulations}

Two subpopulations of organoids with distinct morphology (solid and hollow) are present in cultures from P1, whereas all P2 organoids are morphologically similar [Fig. 7(a)]. The first P1 subpopulation, P1 solid, were generally dense (highly scattering), irregular in morphology, and had core regions with lower ORR values than the periphery. The second P1 subpopulation, P1 hollow, were less dense (lower scattering), had a spherical morphology, and had core regions with higher ORR values than the outer regions. In addition, the ORR values of P1 hollow PCOs were generally higher than $\mathrm{P} 1$ solid $\mathrm{PCO}$, driven by higher $\mathrm{NAD}(\mathrm{P}) \mathrm{H}$ intensity. $\mathrm{P} 2$ appeared to have one type of organoid morphology that was qualitatively similar to P1 solid: dense, irregular in morphology, and with a core region with a low ORR compared to the periphery. The differences in the internal structure of P1 solid and P1 hollow PCOs were confirmed by confocal microscopy of P1 solid and P1 hollow PCOs stained for epithelial cell adhesion molecule (EpCAM, Fig. S3 in the Supplemental Materials). By qualitative examination, 39 P1 solid, 9 P1 hollow, and 35 P2 PCOs were identified.

PCA was applied to the 24 morphological and metabolic variables (Table S1 in the Supplemental Materials) for all pretreatment PCOs to determine if these variables could cluster PCOs by P1 solid, P1 hollow, and P2. A plot of the loading vectors [Fig. 7(b)] shows the contribution of each of the pretreatment variables on the first two PCs. The length of the vector corresponds to the magnitude of the contribution of a specific variable to the first two PCs. The angle between vectors as well as the angle between each vector and the PC axes indicates 
the level of correlation. The colors indicate which are metabolic or morphological variables. Only the 12 variables that have the largest contributions to the first two PCs were displayed for clarity. Figure 7(c) shows the organoid-level data projected onto the PC1-PC2 basis, with clear clustering of subpopulations based on P1 solid, P1 hollow, and P2. Notably, P1 hollow was correlated with metabolic variables, clustering in the lower left quadrant apart from the P1 and P2 clusters [Figs. 7(b) and 7(c)]. The same pretreatment PC1-PC2 basis was used to plot PCOs at 24- and 48-h posttreatment. At 24-h posttreatment, smaller variability is observed within and between clusters compared to pretreatment variability [Fig. 7(d)], and cluster variability increased at $48 \mathrm{~h}$ [Fig. 7(e)].

\section{Discussion}

Functional drug screens using PCOs, which reflect the heterogeneity and drug sensitivity of in vivo tumors, could be used to predict the most effective treatment for a patient and identify novel drug candidates during drug development. ${ }^{5,6,8,10,12}$ However, current methods for evaluating drug response in PCOs are destructive, ignore organoid-level heterogeneity, provide limited metrics of response, or lack throughput. Cell viability assays (e.g., tetrazolium and luciferase) measure metabolic function as a surrogate for the number of viable cells, but use reagents with long-term toxicity, consume the sample, require genetic manipulation, or do not capture heterogeneity. ${ }^{65-68}$ Histology provides cell- and organoid-level structural and molecular information, but requires slow, destructive sample processing. ${ }^{15}$ Standard molecular biology techniques (e.g., RNA-seq, qPCR, and Western blots) can capture treatment-induced changes in protein and RNA levels, but are destructive and require the pooling of many PCOs to yield enough cell material. ${ }^{15}$ Organoid diameter measurements from bright-field images can assess the same PCOs over time, but only provide a single measure of response (i.e., change in organoid area), and these measurements are often performed manually, introducing user bias. ${ }^{11,47}$ Diameter measurements are also limited because changes in PCO diameter or morphology often lag behind functional changes. ${ }^{69-72}$

Here we provide the first demonstration of wide-field one-photon redox imaging for functional assessment of drug response in PCOs. Redox imaging improves on existing tools for PCO screening as it is non-destructive, rapid, and label-free, requiring no additional dyes or reagents. Performing redox imaging in a wide-field one-photon epifluorescence configuration is well suited for high-throughput applications because of simple, widely available instrumentation, large field-of-view, micron-scale resolution, and fast acquisition. However, wide-field onephoton epifluorescence microscopy lacks optical sectioning, limiting its ability to resolve cellular level detail within scattering samples like organoids. The lack of optical sectioning can also be beneficial for high-throughput PCO screening as it captures an integrated response from the entire organoid and is less sensitive to shifts in axial position. Although wide-field one-photon redox imaging does not provide a readout at the cell-level, the organoid-level readout may better represent the response of a patient's tumor as multiple organoids captures more cells for analysis.

New image analysis methods are needed for wide-field one-photon redox imaging that can resolve drug response in heterogenous PCO populations. Specifically, image segmentation and tracking methods are needed to quantify the drug response of each PCO over a treatment time course. These methods enable quantitative measures of the drug response (e.g., changes in organoid morphology and ORR) of each PCO with respect to its matched pretreatment values. Statistical analysis techniques, such as mixed-effect models, can leverage these longitudinal data to account for organoid-level heterogeneity and provide improved sensitivity to drug-induced changes in redox imaging variables. This study shows that wide-field one-photon redox imaging provides automated assessment of multiple quantitative variables at the organoid-level that capture drug response and separate PCO subpopulations based on phenotype. A key result of this technical development paper is a framework to acquire robust redox imaging data that links values within the same PCO across multiple time points using a standard epifluorescence microscope. This proof-of-concept study used samples from two patients, and additional studies are needed to confirm that the specific observations apply to other PCOs and treatments. These methods can be adopted by non-experts to achieve reproducible data of drug response in 
PCOs using accessible instrumentation, while accounting for pretreatment variability between PCOs to yield meaningful drug response data.

This was the first study to perform wide-field one-photon redox imaging of PCOs, so the relationships between the organoid-level morphological and metabolic variables were unknown. The relationship between organoid size and ORR was of interest as PCOs cultured over long periods of time form a characteristic core of dead cells with a lower ORR. The lower ORR is likely due to increased flavin production (riboflavin, flavin mononucleotide, and FAD) during the cellular response to cytotoxic stress, suggesting the ORR $[\mathrm{NAD}(\mathrm{P}) \mathrm{H} /(\mathrm{FAD}+\mathrm{NAD}(\mathrm{P}) \mathrm{H})]$ is sensitive to cell death. ${ }^{73,74}$ Interestingly, we found that the mean ORR was independent of organoid area for the PCOs imaged in this study (area range: 0.02 to $0.9 \mathrm{~mm}^{2}$ ). Larger PCOs exhibited higher mean FAD intensity values due to larger dead cores, but this was compensated by increased $\mathrm{NAD}(\mathrm{P}) \mathrm{H}$ levels at the periphery, which we hypothesize is due to the presence of more viable cell material. Further analysis of all variables revealed that the mean ORR is uncorrelated with other measures of organoid morphology, indicating that the ORR provides complementary information to morphology. Each PCO was quantified without background subtraction to limit the effects of variations in the autofluorescence of the culture medium.

PCOs grow stochastically due to the culture technique (single-cell suspension in a 3D matrix) and cellular heterogeneity, resulting in organoids that are unique in size, morphology, and metabolism pretreatment and posttreatment. ${ }^{75,76}$ Previous studies of drug response in PCOs have only assessed response at single time point or used different organoids for each time point, both of which only capture a snapshot of the PCO population. Automated single-organoid tracking enables measurement of organoid-level drug response over multiple time points. Drug response was assessed by measuring organoid-level changes in the ORR and growth. Our results show that drug response leads to a decreased ORR and growth rate for PCOs compared to control at multiple time points (Figs. 4 and 5), which is consistent with results from previous studies. Temporally, changes in the ORR precede changes in the organoid growth rate, which indicates that the ORR provides an early measurement of PCO drug response. ${ }^{43,77,78}$ Tracking organoids over time also overcomes issues with variability in the numbers and sizes of PCOs in each well. Statistical techniques such as mixed-effect models can use these time-course data to account for organoid-level heterogeneity and provide improved sensitivity to drug response. ${ }^{63,79}$ Our results show that wide-field one-photon redox imaging can determine drug response in PCOs, and that single-organoid tracking improves sensitivity to treatment-induced changes in redox imaging variables (Fig. 6).

PCOs generated from P1 exhibited two distinct phenotypes, P1 solid and P1 hollow, which were identified by visual inspection and confirmed by multivariate analysis (PCA). The fluidfilled hollow morphology of P1 hollow PCOs are consistent with cystic morphologies seen in other papers using colorectal PCOs. ${ }^{80}$ As these phenotypes are stable over multiple passages, these two subpopulations likely reflect the in vivo heterogeneity of the patient's tumor and are not due to mutations gained in vitro. The loading vectors [Fig. 7(b)] appear to confirm that the metabolic and morphological variables are orthogonal, which demonstrates the information gained by redox imaging and is consistent with the limited correlations between organoid metabolism and morphology [Fig. 3(d)]. Although the loadings of the morphological variables were larger, both the metabolic variables and morphological variables were needed to show clustering of P1 hollow from P1 solid and P2. As the P1 hollow subpopulation comprised only 19\% of all P1 PCOs and were not evenly distributed among the five treatment groups, stratification of the response of each subpopulation was not attempted. Additional analysis was performed to quantify the spatial distribution of autofluorescence within organoids from each PCO subtype (Fig. S4 in the Supplemental Materials). The intensity of NAD(P)H and FAD decreases and increases, respectively, in the core compared to the rim of solid organoids. Prior studies have shown that organoids with this solid phenotype have an apoptotic core identified using immunofluorescence of cleaved caspase $3{ }^{44}$ Apoptotic cells exhibit high FAD intensity, resulting in a low ORR at the core of solid organoids (Fig. S4 in the Supplemental Materials). ${ }^{73,74}$ Conversely, the intensity of $\mathrm{NAD}(\mathrm{P}) \mathrm{H}$ and FAD increases and decreases, respectively, in the core compared to the rim of hollow organoids. This results in a high ORR in the core of hollow organoids, which do not appear to have an apoptotic core (Fig. S4 in the Supplemental Materials). 
This study demonstrates a framework for performing wide-field one-photon redox imaging of PCOs and single-organoid tracking that accounts for pretreatment heterogeneity in organoid morphology and metabolism for robust drug response measurements. Wide-field one-photon redox imaging is an easily scalable and accessible technology that provides both metabolic and morphological information for characterizing PCO populations. We believe that these validated approaches will enable broad dissemination of wide-field one-photon redox imaging for screening of PCOs, with applications in drug development and clinical treatment planning.

\section{Disclosures}

Authors have no relevant financial interests in the manuscript and no other potential conflicts of interest to disclose.

\section{Acknowledgments}

We would like to thank Tongcheng Qian for providing the confocal microscopy images. We also would like to thank Tiffany Heaster, Rupsa Datta, Peter Favreau, and Maryse Lapierre-Landry for their valuable feedback on this manuscript. This work was supported by the National Science Foundation (No. CBET-1642287), Stand Up to Cancer (No. SU2C-AACR-IG-08-16), the National Institutes of Health (Nos. R01 CA185747, R01 CA185747, and R37 CA226526), and the University of Wisconsin Carbone Cancer Center (Support Grant No. P30 CA014520).

\section{References}

1. E. Fountzilas and A. M. Tsimberidou, "Overview of precision oncology trials: challenges and opportunities," Expert Res. Clin. Pharmacol. 11(8), 797-804 (2018).

2. V. Prasad, "Perspective: the precision-oncology illusion," Nature 537(7619), S63-S63 (2016).

3. I. F. Tannock and J. A. Hickman, "Limits to personalized cancer medicine," N. Engl. J. Med. 375(13), 1289-1294 (2016).

4. A. Letai, "Functional precision cancer medicine-moving beyond pure genomics," Nat. Med. 23(9), 1028-1035 (2017).

5. N. Sachs et al., "A living biobank of breast cancer organoids captures disease heterogeneity," Cell 172(1-2), 373-386.e10 (2018).

6. G. Vlachogiannis et al., "Patient-derived organoids model treatment response of metastatic gastrointestinal cancers," Science 359(6378), 920-926 (2018).

7. Y. Saito et al., "Establishment of patient-derived organoids and drug screening for biliary tract carcinoma," Cell Rep. 27(4), 1265-1276.e4 (2019).

8. H. Tiriac et al., "Organoid profiling identifies common responders to chemotherapy in pancreatic cancer," Cancer Discovery 8(9), 1112 (2018).

9. D. Schumacher et al., "Heterogeneous pathway activation and drug response modelled in colorectal-tumor-derived 3D cultures," PLoS Genet. 15(3), e1008076 (2019).

10. N. Sasaki and H. Clevers, "Studying cellular heterogeneity and drug sensitivity in colorectal cancer using organoid technology," Curr. Opin. Genet. Dev. 52, 117-122 (2018).

11. C. A. Pasch et al., "Patient-derived cancer organoid cultures to predict sensitivity to chemotherapy and radiation," Clin. Cancer Res. 25(17), 5376-5387 (2019).

12. O. Kopper et al., "An organoid platform for ovarian cancer captures intra- and interpatient heterogeneity," Nat. Med. 25(5), 838-849 (2019).

13. J. T. Sharick et al., "Cellular metabolic heterogeneity in vivo is recapitulated in tumor organoids," Neoplasia 21(6), 615-626 (2019).

14. K. Y. Chen et al., "Single-cell transcriptomics reveals heterogeneity and drug response of human colorectal cancer organoids," in Annu. Int. Conf. IEEE Eng. Med. Biol. Soc., pp. 2378-2381 (2018).

15. S. H. Lee et al., "Tumor evolution and drug response in patient-derived organoid models of bladder cancer," Cell 173(2), 515-528.e17 (2018). 
Gil, Deming, and Skala: Patient-derived cancer organoid tracking with wide-field one-photon...

16. A. Luengo, D. Y. Gui, and M. G. Vander Heiden, "Targeting metabolism for cancer therapy," Cell Chem. Biol. 24(9), 1161-1180 (2017).

17. D. A. Gil et al., "Redox imaging and optical coherence tomography of the respiratory ciliated epithelium," J. Biomed. Opt. 24(1), 010501 (2019).

18. A. J. Walsh and M. C. Skala, "Optical metabolic imaging quantifies heterogeneous cell populations," Biomed. Opt. Express 6(2), 559-573 (2015).

19. A. Walsh et al., "Optical imaging of metabolism in HER2 overexpressing breast cancer cells," Biomed. Opt. Express 3(1), 75-85 (2012).

20. A. Varone et al., "Endogenous two-photon fluorescence imaging elucidates metabolic changes related to enhanced glycolysis and glutamine consumption in precancerous epithelial tissues," Cancer Res. 74(11), 3067-3075 (2014).

21. M. C. Skala et al., "In vivo multiphoton microscopy of NADH and FAD redox states, fluorescence lifetimes, and cellular morphology in precancerous epithelia," Proc. Natl. Acad. Sci. U. S. A. 104(49), 19494-19499 (2007).

22. M. C. Skala et al., "Longitudinal optical imaging of tumor metabolism and hemodynamics," J. Biomed. Opt. 15(1), 011112 (2010).

23. M. Skala and N. Ramanujam, "Multiphoton redox ratio imaging for metabolic monitoring in vivo," Methods Mol. Biol. 594, 155-162 (2010).

24. A. T. Shah et al., "In vivo autofluorescence imaging of tumor heterogeneity in response to treatment," Neoplasia 17(12), 862-870 (2015).

25. A. T. Shah et al., "Optical metabolic imaging of treatment response in human head and neck squamous cell carcinoma," PLoS One 9(3), e90746 (2014).

26. H. Xu et al., "Calibration of CCD-based redox imaging for biological tissues," Proc. SPIE 7262, 72622F (2009).

27. M. Ranji et al., "Fluorescence spectroscopy and imaging of myocardial apoptosis," J. Biomed. Opt. 11(6), 064036 (2006).

28. H. N. Xu et al., "Optical redox imaging indices discriminate human breast cancer from normal tissues," J. Biomed. Opt. 21(11), 114003 (2016).

29. K. Alhallak et al., "Optical imaging of radiation-induced metabolic changes in radiationsensitive and resistant cancer cells," J. Biomed. Opt. 22(6), 060502 (2017).

30. J. D. Jones et al., "In vivo multiphoton microscopy detects longitudinal metabolic changes associated with delayed skin wound healing," Commun. Biol. 1, 198-198 (2018).

31. O. I. Kolenc and K. P. Quinn, "Evaluating cell metabolism through autofluorescence imaging of NAD(P)H and FAD," Antioxid. Redox Signaling 30(6), 875-889 (2019).

32. M. MasoudiMotlagh et al., "Optical cryoimaging of mitochondrial redox state in bronchopulmonary-dysplasia injury models in mice lungs," Quant. Imaging Med. Surg. 5(1), 159-162 (2015).

33. J. H. Ostrander et al., "Optical redox ratio differentiates breast cancer cell lines based on estrogen receptor status," Cancer Res. 70(11), 4759-4766 (2010).

34. K. P. Quinn et al., "Quantitative metabolic imaging using endogenous fluorescence to detect stem cell differentiation," Sci. Rep. 3, 3432 (2013).

35. Y. Wen et al., "Optical redox imaging detects the effects of DEK oncogene knockdown on the redox state of MDA-MB-231 breast cancer cells," Mol. Imaging Biol. 21(3), 410-416 (2019).

36. B. Chance et al., "Oxidation-reduction ratio studies of mitochondria in freeze-trapped samples. NADH and flavoprotein fluorescence signals," J. Biol. Chem. 254(11), 4764-4771 (1979).

37. A. A. Heikal, "Intracellular coenzymes as natural biomarkers for metabolic activities and mitochondrial anomalies," Biomark Med. 4(2), 241-263 (2010).

38. I. Georgakoudi and K. P. Quinn, "Optical imaging using endogenous contrast to assess metabolic state," Апnи. Rev. Biomed. Eng. 14, 351-367 (2012).

39. A. T. Shah, T. M. Heaster, and M. C. Skala, "Metabolic imaging of head and neck cancer organoids," PLoS One 12(1), e0170415 (2017).

40. T. M. Cannon et al., "High-throughput measurements of the optical redox ratio using a commercial microplate reader," J. Biomed. Opt. 20(1), 010503 (2015). 
Gil, Deming, and Skala: Patient-derived cancer organoid tracking with wide-field one-photon...

41. A. J. Walsh et al., "Optical metabolic imaging identifies glycolytic levels, subtypes, and early-treatment response in breast cancer," Cancer Res. 73(20), 6164-6174 (2013).

42. J. T. Sharick et al., "Metabolic heterogeneity in patient tumor-derived organoids by primary site and drug treatment," Front. Oncol. 10, 553 (2020).

43. A. J. Walsh et al., "Quantitative optical imaging of primary tumor organoid metabolism predicts drug response in breast cancer," Cancer Res. 74(18), 5184-5194 (2014).

44. P. F. Favreau et al., "Label-free redox imaging of patient-derived organoids using selective plane illumination microscopy," Biomed. Opt. Express 11(5), 2591-2606 (2020).

45. S. Mehrvar et al., "Fluorescence imaging of mitochondrial redox state to assess diabetic wounds," IEEE J. Transl. Eng. Health Med. 7, 1-9 (2019).

46. N. Phan et al., "A simple high-throughput approach identifies actionable drug sensitivities in patient-derived tumor organoids," Commun. Biol. 2(1), 78 (2019).

47. S. L. Fricke et al., "MTORC1/2 inhibition as a therapeutic strategy for PIK3CA mutant cancers," Mol. Cancer Ther. 18(2), 346-355 (2019).

48. T. Zhan, N. Rindtorff, and M. Boutros, "Wnt signaling in cancer," Oncogene 36(11), 1461-1473 (2017).

49. J. L. Way, "Cyanide intoxication and its mechanism of antagonism," Annu. Rev. Pharmacol. Toxicol. 24, 451-481 (1984).

50. D. Zhang et al., "2-Deoxy-D-glucose targeting of glucose metabolism in cancer cells as a potential therapy," Cancer Lett. 355(2), 176-183 (2014).

51. S. Dasari and P. B. Tchounwou, "Cisplatin in cancer therapy: molecular mechanisms of action," Eur. J. Pharmacol. 740, 364-378 (2014).

52. B. A. Weaver, "How taxol/paclitaxel kills cancer cells," Mol. Biol. Cell 25(18), 2677-2681 (2014).

53. A. D. Edelstein et al., "Advanced methods of microscope control using $\mu$ Manager software," J. Biol. Methods 1(2), e10 (2014).

54. M. Guizar-Sicairos, S. T. Thurman, and J. R. Fienup, "Efficient subpixel image registration algorithms," Opt. Lett. 33(2), 156-158 (2008).

55. M. Guizar, "Efficient subpixel image registration by cross-correlation," MATLAB Central File Exchange (2020).

56. P. Soille, Morphological Image Analysis: Principles and Applications, 2nd ed., Springer, Berlin, New York (2003).

57. J. Y. Tinevez et al., "TrackMate: an open and extensible platform for single-particle tracking," Methods 115, 80-90 (2017).

58. K. Jaqaman et al., "Robust single-particle tracking in live-cell time-lapse sequences," Nat. Methods 5(8), 695-702 (2008).

59. L. van der Maaten, "drtoolbox: Matlab toolbox for dimensionality reduction" (2019).

60. P. Morel, "Gramm: grammar of graphics plotting in Matlab," J. Open Source Software 3(23), 568 (2018).

61. D. Bates et al., "nlme: linear and nonlinear mixed effects models" (2018).

62. R. V. Lenth, "Least-squares means: the R package lsmeans," J. Stat. Software 69(1), 1-33 (2016).

63. R. D. Gibbons, D. Hedeker, and S. DuToit, "Advances in analysis of longitudinal data," Annu. Rev. Clin. Psychol. 6, 79-107 (2010).

64. X. A. Harrison et al., "A brief introduction to mixed effects modelling and multi-model inference in ecology," PeerJ 6, e4794-e4794 (2018).

65. R. Hamid et al., "Comparison of alamar blue and MTT assays for high through-put screening," Toxicol. In Vitro 18(5), 703-710 (2004).

66. L. Lü et al., "Exocytosis of MTT formazan could exacerbate cell injury," Toxicol. In Vitro 26(4), 636-644 (2012).

67. G. Fotakis and J. A. Timbrell, "In vitro cytotoxicity assays: comparison of LDH, neutral red, MTT and protein assay in hepatoma cell lines following exposure to cadmium chloride," Toxicol. Lett. 160(2), 171-177 (2006).

68. A. van Tonder, A. M. Joubert, and A. D. Cromarty, "Limitations of the 3-(4,5dimethylthiazol-2-yl)-2,5-diphenyl-2H-tetrazolium bromide (MTT) assay when compared to three commonly used cell enumeration assays," BMC Res. Notes 8, 47-47 (2015). 
Gil, Deming, and Skala: Patient-derived cancer organoid tracking with wide-field one-photon...

69. L. Yang et al., "Characterizing optical coherence tomography speckle fluctuation spectra of mammary organoids during suppression of intracellular motility," Quant. Imaging Med. Surg. 10(1), 76-85 (2020).

70. S. Hao et al., "Biodynamic imaging for phenotypic profiling of three-dimensional tissue culture," J. Biomed. Opt. 22(1), 016007 (2017).

71. G. Narayanan et al., "Intracellular Doppler spectroscopy detects altered drug response in SKOV3 tumor spheroids with silenced or inhibited P-glycoprotein," Biochem. Biophys. Res. Commun. 514(4), 1154-1159 (2019).

72. Z. Li et al., "Intracellular optical Doppler phenotypes of chemosensitivity in human epithelial ovarian cancer," Sci. Rep. 10(1), 17354 (2020).

73. J. Xu and W. Ying, "Increased green autofluorescence is a marker for non-invasive prediction of $\mathrm{H}_{2} \mathrm{O}_{2}$-induced cell death and decreases in the intracellular ATP of HaCaT cells," bioRxiv 298075 (2018).

74. J. Surre et al., "Strong increase in the autofluorescence of cells signals struggle for survival," Sci. Rep. 8(1), 12088 (2018).

75. M. A. Borten et al., "Automated brightfield morphometry of 3D organoid populations by OrganoSeg," Sci. Rep. 8(1), 5319 (2018).

76. S. M. Tirier et al., "Pheno-seq-linking visual features and gene expression in 3D cell culture systems," Sci. Rep. 9(1), 12367 (2019).

77. Y. Jung et al., "Longitudinal, label-free, quantitative tracking of cell death and viability in a 3D tumor model with OCT," Sci. Rep. 6, 27017 (2016).

78. Y. Huang et al., "Optical coherence tomography detects necrotic regions and volumetrically quantifies multicellular tumor spheroids," Cancer Res. 77(21), 6011-6020 (2017).

79. G. Verbeke et al., "The analysis of multivariate longitudinal data: a review," Stat. Methods Med. Res. 23(1), 42-59 (2014).

80. S. M. H. Kashfi et al., "Morphological alterations of cultured human colorectal matched tumour and healthy organoids," Oncotarget 9(12), 10572-10584 (2018).

Biographies of the authors are not available. 